OPEN ACCESS

Edited by:

Kun $\mathrm{Hu}$,

Harvard Medical School,

United States

Reviewed by:

Chenlu Gao,

Brigham and Women's Hospital and

Harvard Medical School,

United States

Chaur-Jong Hu,

Taipei Medical University, Taiwan

Akihiro Shindo,

Mie University Hospital, Japan

*Correspondence:

Hung-Chieh Chen

hungchiehchen@gmail.com

Ting-Bin Chen

sophiebeen@gmail.com

†These authors have contributed equally to this work

Specialty section:

This article was submitted to Dementia and Neurodegenerative

Diseases,

a section of the journal

Frontiers in Neurology

Received: 26 July 2021 Accepted: 08 October 2021 Published: 12 November 2021

Citation:

Lin C-Y, Jhan S-R, Lee W-J, Chen $P$ - L, Chen J-P, Chen H-C and Chen T-B (2021) Imaging Markers of

Subcortical Vascular Dementia in Patients With Multiple-Lobar Cerebral Microbleeds.

Front. Neurol. 12:747536

doi: 10.3389/fneur.2021.747536

\section{Imaging Markers of Subcortical Vascular Dementia in Patients With Multiple-Lobar Cerebral Microbleeds}

\author{
Chia-Yen Lin ${ }^{1 \dagger}$, Song-Ru Jhan ${ }^{2 \dagger}$, Wei-Ju Lee ${ }^{1,3,4}$, Po-Lin Chen ${ }^{1,4}$, Jun-Peng Chen ${ }^{5}$, \\ Hung-Chieh Chen ${ }^{2,4 *}$ and Ting-Bin Chen ${ }^{1,3,6 *}$ \\ ${ }^{1}$ Department of Neurology, Neurological Institute, Taichung Veterans General Hospital, Taichung, Taiwan, ${ }^{2}$ Division of \\ Neuroradiology, Department of Radiology, Taichung Veterans General Hospital, Taichung, Taiwan, ${ }^{3}$ Dementia Center, \\ Taichung Veterans General Hospital, Taichung, Taiwan, ${ }^{4}$ School of Medicine, National Yang-Ming Chiao Tung University, \\ Taipei, Taiwan, ${ }^{5}$ Biostatistics Task Force of Taichung Veterans General Hospital, Taichung, Taiwan, ${ }^{6}$ Department of Applied \\ Cosmetology, Hungkuang University, Taichung, Taiwan
}

Background and Purpose: Small vessel disease (SVD) imaging markers are related to ischemic and hemorrhage stroke and to cognitive dysfunction. This study aimed to clarify the relationship between SVD imaging markers and subcortical vascular dementia in severe SVD burden.

Methods: A total of 57 subjects with multiple lobar cerebral microbleeds (CMBs) and four established SVD imaging markers were enrolled from the dementia and stroke registries of a single center. Visual rating scales that are used to semi-quantify SVD imaging changes were analyzed individually and compositely to make correlations with cognitive domains and subcortical vascular dementia.

Results: Dementia group had higher subcortical and total white matter hyperintensities (WMHs) and SVD composite scores than non-dementia group. Individual imaging markers correlated differently with one another and had distinct cognitive correlations. After adjusting for demographic factors, multivariate logistic regression indicated associations of subcortical WMHs (odds ratio [OR] 2.03, Cl 1.24-3.32), total WMHs (OR 1.43, Cl 1.09-1.89), lacunes (OR 1.18, Cl 1.02-1.35), cerebral amyloid angiopathy-SVD scores (OR 2.33, $\mathrm{Cl} 1.01-5.40), \mathrm{C}_{1}$ scores (imaging composite scores of $\mathrm{CMB}$ and $\mathrm{WMH}$ ) (OR 1.41, $\mathrm{Cl} 1.09-1.83$ ), and $\mathrm{C}_{2}$ scores (imaging composite scores of $\mathrm{CMB}, \mathrm{WMH}$, perivascular space, and lacune) (OR 1.38, $\mathrm{Cl}$ 1.08-1.76) with dementia.

Conclusions: SVD imaging markers might have differing associations with cognitive domains and dementia. They may provide valuable complementary information in support of personalized treatment planning against cognitive impairment, particularly in patients with a heavy SVD load.

Keywords: hypertensive angiopathy, amyloid angiopathy, lacune, microbleed, white matter hyperintensities 


\section{INTRODUCTION}

Small vessel disease (SVD) is characterized by a variety of cerebral parenchymal lesions that can be detected by neuroimaging, including cerebral microbleeds (CMBs), white matter hyperintensities (WMHs), lacunes, and enlargements of the perivascular space (PVSEs) (1). These SVD-related MRI markers have been associated with ischemic and hemorrhagic stroke and dementia (1), and these features have also been linked to vascular risk factors, cognitive dysfunction, depression, and epilepsy $(2,3)$. These markers often co-occur, together constituting a composite SVD burden. Several studies using composite scoring scales of the salient SVD-related MRI signatures have demonstrated a negative impact of global SVD burden on cognition, functional ability, gait, and balance (4-8).

Cerebral microbleeds increase the risk of spontaneous intracerebral hemorrhage (ICH), ischemic stroke, cognitive impairment, and gait disturbance $(9,10)$. Two main sporadic forms of SVD present with CMBs: hypertensive arteriopathy (HA) and cerebral amyloid angiopathy (CAA). The former affects the small perforating end arteries of deep gray nuclei and deep WM. The latter is characterized by progressive amyloid-beta deposition in the tunica media and adventitia of small cortical vessels overlying the leptomeninges and gray-WM junction. The topographical CMB distribution could be utilized to identify the underlying SVD pathogenesis. Deep and infratentorial (i.e., nonlobar) CMBs are indicative of HA, whereas strictly lobar CMBs suggest CAA (11). Multiple lobar CMBs, like strictly lobar CMBs, could suggest CAA pathology (12). A mix of lobar with nonlobar CMBs can occur in HA, but a HA-CAA synergism could also be responsible for $\mathrm{CMB}$ pathogenesis, which implies an SVD spectrum $(10,11,13)$.

White matter hyperintensities are predictive of stroke risk and have been associated with declining global cognition, executive function, and processing speed, as well as with dementia, depression, and death due to cardiovascular causes (14). Periventricular WMHs and deep WMHs have distinct pathophysiologies and clinical significances (15). Major risk factors for WMH development are advanced age, hypertension, and cardiovascular disease. The underlying pathology of WMHs in subcortical vascular dementia involves demyelination and axon loss due to SVD ischemia (16).

The PVS is the fluid-containing space surrounding blood vessels, as they course from the subarachnoid space into the brain (17). PVSEs may result from obstructed drainage (17). Basal ganglia (BG) PVSEs have been associated with advanced age, hypertension, and stroke, whereas centrum semiovale (CS) PVSEs have been associated with CAA or a mixed hypertensive/CAA pathophysiology $(18,19)$.

Although SVD changes are commonly seen in routine MRIs, little is known about their cognitive contributions in patients with multiple lobar CMBs. We used a validated CAA-SVD scoring system, showing close association with the severity of CAA-related vasculopathic changes at autopsy, to represent the underlying CAA load in the brain in our cohort $(4,20)$. Additionally, we developed two comparable ordinal scores based on the four established MRI markers of SVD to compile the overall brain burden. The primary aim of this work was to investigate associations of individual regional imaging markers and composite scores with cognitive performance and dementia risk in patients with multiple lobar CMBs and coexisting all four MRI features of SVD.

\section{METHODS}

\section{Subjects}

We identified patients with dementia or acute stroke from the dementia care database and stroke registry at Taichung Veterans General Hospital from January 2017 to March 2020. The institutional review board and the ethics committee of Taichung Veterans General Hospital approved the data collection protocol. Informed consent was waived by ethics committee of Taichung Veterans General Hospital.

We first considered all subjects over 55 years old diagnosed with subcortical vascular dementia, acute symptomatic lacunar stroke, or acute symptomatic spontaneous lobar ICH. Those with all four features of SVD (i.e., CMBs, WMHs, PVSEs, and lacunes) and multiple lobar CMBs visible on MRI were enrolled. Multiple $(\geq 2)$ lobar was defined as involving more than one $(\geq 2)$ frontal, parietal, temporal, or occipital lobes of right or left hemisphere, with or without CMBs in a deep or infratentorial region. The exclusion criteria were: age $<55$ years; diagnosis with an uncertain dementia; acute ischemic stroke due to causes other than small-vessel occlusion; ICH due to head trauma; hemorrhagic transformation of a stroke; arteriovenous malformation; hemorrhagic tumor; recipient of anticoagulant therapy; and vasculitis.

Two well-trained licensed nurse case managers conducted registration management, including collection of a brief history, examination findings, age, sex, body mass index, medical comorbidities (including cardiovascular and metabolic diseases), and cigarette and alcohol usage. The Charlson comorbidity index (CCI), which contains 19 weighted comorbidities, was also gathered to determine the overall systemic health of each individual (21). Cognitive performance in the subjects with cognitive decline was assessed by neuropsychologists using 12 -item word recall test (total correct trials $1-3$, and 15-min delayed free recall) $(22,23)$, estimated Mini-Mental State Examination (MMSE) converted from Cognitive Abilities Screening Instrument $(24,25)$, Montreal Cognitive Assessment (MoCA) (26), and Clinical Dementia Rating scale (27). Cognitive performance was documented as well as the contents of diagnostic workups, dementia disorder type, pharmacological and non-pharmacological treatments, and support for the patient from the county.

\section{Clinical Diagnosis and Grouping}

Medical records and registry data were reviewed in detail. Subjects were dichotomized into dementia group and non-dementia group. Dementia diagnoses were made in accordance with standard criteria based on a clinical interview, functional assessments, neurological examinations, neuropsychological screening, MRI, and laboratory studies (28). Probable Alzheimer's disease was diagnosed when 
subjects meet the clinical criteria proposed by the National Institute of Neurological and Communicative Disorders and Stroke/Alzheimer's Disease and Related Disorders Association (29) and the National Institute on Aging/Alzheimer's Association workgroup (30). Diagnoses of vascular dementia, frontotemporal dementia, and dementia with Lewy bodies were made according to their respective criteria (31-34).

Acute stroke was confirmed by board-certified neurologists and/or neurosurgeons based on clinical symptoms, neurological signs, and brain MRI findings. Ischemic stroke subtypes were classified into five etiological categories in accordance with the Trial of ORG 10172 in Acute Stroke Treatment (TOAST) rubric (35).

\section{Brain MRI}

All subjects were scanned in a 1.5-T MRI scanner (MAGNETOM Aera, Siemens Healthcare, Erlangen, Germany). MRI scanning was done within 3 months after cognitive assessment in the patients with dementia and in the setting of acute clinical event in the patients with acute stroke. MRI was performed according to a standardized protocol inclusive of the following sequences: axial spin echo (SE) T1-weighted images (T1WI) [repetition time $(\mathrm{TR}) /$ echo time $(\mathrm{TE})=550 / 8.9 \mathrm{~ms}$, Field-of-View $(\mathrm{FOV})=$ $230^{*} 230 \mathrm{~mm}^{2}$, matrix size $=320^{*} 256$, slice thickness $=6 \mathrm{~mm}$ ], axial turbo-SE (TSE) T2-weighted images (T2WI) $(\mathrm{TR} / \mathrm{TE}=$ $3,500 / 103 \mathrm{~ms}$, FOV $=230 * 208 \mathrm{~mm}^{2}$, matrix size $=512 * 384$, slice thickness $=6 \mathrm{~mm}$ ), axial fluid-attenuated inversion recovery (FLAIR) $\left(\mathrm{TR} / \mathrm{TE} / \mathrm{TI}=9,000 / 86 / 2,500 \mathrm{~ms}, \mathrm{FOV}=230^{*} 201 \mathrm{~mm}^{2}\right.$, matrix size $=320 * 240$, slice thickness $=6 \mathrm{~mm}$ ), axial diffusion weighted images (DWI) $\left(\mathrm{TR} / \mathrm{TE}=6,300 / 89 \mathrm{~ms}, \mathrm{FOV}=230^{*} 230\right.$ $\mathrm{mm}^{2}$, matrix size $=192^{*} 192$, slice thickness $=6 \mathrm{~mm}$ ), oblique coronal TSE T2-weighted images (T2WI) for temporal lobe $\left(\mathrm{TR} / \mathrm{TE}=4,000 / 83 \mathrm{~ms}, \mathrm{FOV}=180^{*} 180 \mathrm{~mm}^{2}\right.$, matrix size $=384^{*} 307$, slice thickness $=3 \mathrm{~mm}$ ), axial $3 \mathrm{D}$ time-of-flight magnetic resonance angiography (3D-TOF-MRA) $(\mathrm{TR} / \mathrm{TE}=$ $24 / 7 \mathrm{~ms}, \mathrm{FOV}=180^{*} 180 \mathrm{~mm}^{2}$, matrix size $=256^{*} 218$, flip angle $=25^{\circ}$, slice thickness $=0.5 \mathrm{~mm}$, slabs $=4$ slices per slab $=52$ ), and axial susceptibility-weighted MRI (SWI) (TR/TE $=49 / 40 \mathrm{ms,}$ FOV $=230^{*} 186 \mathrm{~mm}^{2}$, flip angle $=15^{\circ}$, matrix size $=288^{*} 230$, slice thickness $=2 \mathrm{~mm}$ ). The total scan time is about $35 \mathrm{~min}$.

\section{Visual Rating}

Two independent experienced neuroradiologists rated the MRI data. CMBs, WMHs, and PVSEs were defined according to the Standards for Reporting Vascular changes on Neuroimaging (STRIVE) consensus (36). CMBs were recognized as $\leq 10 \mathrm{~mm}$ homogeneous-rounded hypointense lesions on SWI. CMBs were counted in lobar, deep, and infratentorial regions based on the Microbleed Anatomical Rating Scale (MARS) (37). Ill-defined hyperintensities $\geq 5 \mathrm{~mm}$ on T2WI and FLAIR images were recognized as WMHs. WMHs detected in five brain regions (frontal lobe, parietal-occipital lobe, temporal lobe, BG, and infratentorial region) in the hemisphere of more severe $\mathrm{WMH}$ were graded according to the Age-Related White Matter Change (ARWMC) scale (range, 0-3) (38). The ARWMC scale scores for each lobe aggregated by hemisphere were designated as subcortical ARWMC scale. All ARWMC scores in the five brain regions were combined to calculate total ARWMC score that reflected WMH severity of the entire hemisphere. The ARWMC scale has been shown to correlate with WMH volume $(39,40)$. Periventricular and deep WMHs were graded according to a modified Fazekas scale (range, 0-3) (41). The PVS regions were considered enlarged when spaces measuring $>3 \mathrm{~mm}$ were visible with signal intensity similar to cerebrospinal fluid on T2WI (36). The PVSEs were counted and rated semi-quantitatively in the BG and CS as follows: 0, no PVSEs; 1, 1-10; 2, 11-20; 3, 21-40; and 4, >40 PVSEs (42). We counted ICHs and lacunes visible on FLAIR and SWI sequences. Cortical superficial siderosis (cSS) was identified as a curvilinear hypointensity following the gyral surface on SWI. There was high inter-rater reliability, and the intra-class correlation coefficients between the raters for MARS total score and ARWMC total score were between 0.98 and 0.99 .

\section{SVD Composite Scoring}

We adopted a validated CAA-SVD scoring reflexive of total CAA pathological burden in the brain $(4,20)$. Briefly, each marker category was scored on a $0-6$ scale. Lobar CMBs were scored 1 point if there were $2-4 \mathrm{CMBs}$ or 2 points if there were $\geq 5$. Focal cSS was scored 1 and disseminated cSS was scored 2 points. One point (each) was scored for $\geq 20$ CS PVSEs and WMHs with periventricular Fazekas score $\geq 3$ or a deep Fazekas score $\geq 2$. We generated a composite score $1\left(\mathrm{C}_{1}\right)$ comprising the total MARS grade (1 point for $2-4 \mathrm{CMBs} ; 2$ points for $\geq 5 \mathrm{CMBs}$ ) and the total ARWMC score. A composite score $2\left(\mathrm{C}_{2}\right)$ consisted of $\mathrm{C} 1$, BG PVSE, and lacune amount. One point (each) was scored for $\geq$ 20 BG PVSEs or $\geq 5$ lacunes. $C_{2}$ score was equivalent to the global cerebral SVD score developed to represent total SVD burden of both HA and CAA pathologies (20).

\section{Statistical Analysis}

Analyses were performed in Statistical Package for the Social Sciences (SPSS) version 22.0 for Windows (SPSS Inc., Chicago, IL). Two-tailed $p<0.05$ were considered significant. Differences in continuous/categorical variables between patients with vs. without subcortical vascular dementia were detected with MannWhitney $U$-test/Chi-square tests. We generated neurocognitive numeric composite $\mathrm{z}$-scores by calculating individual $\mathrm{z}$-scores for each test and then averaging them across the cognitive test set. The constituents of the composite $\mathrm{z}$-scores were as follows: attention [Serial 7s on the MMSE and sustained attention task and forward and backward digit span on the MoCA], shortterm memory (registration) [total number of items remembered over three trials on the 12-item memory test, immediate memory task on the MMSE], short-term memory (recall) [15-min delayed recall on the 12-item memory test, delayed memory recall task on the MMSE and MoCA]; orientation [orientation tests on the MMSE and MoCA]; language [confrontation naming task, repetition of two syntactically complex sentences, writing, and fluency task on the MMSE and MoCA]; visuoexecutive function [pentagon test on the MMSE and clock-drawing task and a three-dimensional cube copy on the MoCA]. Spearman's rank coefficients were calculated to determine the correlation of imaging variables with each other and constituent cognitive domains. To investigate the association of imaging variables with 
TABLE 1 | Demographic and clinical characteristics of this cohort, $N=57$.

\begin{tabular}{lccc}
\hline Characteristic & $\begin{array}{c}\text { Dementia } \\
(\mathbf{N}=\mathbf{1 2})\end{array}$ & $\begin{array}{c}\text { Non-dementia } \\
(\mathbf{N}=\mathbf{4 5})\end{array}$ & $\boldsymbol{P}$ \\
\hline Sex, female, $N(\%)$ & $4(33.3 \%)$ & $13(28.9 \%)$ & 0.737 \\
Age, years & $74.5(71.5-82.3)$ & $72.0(63.5-82.5)$ & 0.337 \\
Smoking, $N(\%)$ & $6(50.0 \%)$ & $16(35.6 \%)$ & 0.506 \\
Hypertension, $N$ (\%) & $10(83.3 \%)$ & $41(91.1 \%)$ & 0.596 \\
Diabetes mellitus, $N(\%)$ & $3(25.0 \%)$ & $14(31.1 \%)$ & 1.000 \\
Hyperlipidemia, $N(\%)$ & $4(33.3 \%)$ & $24(53.3 \%)$ & 0.365 \\
Peripheral or cardiac & $2(16.7 \%)$ & $11(24.4 \%)$ & 0.713 \\
vasculopathy ${ }^{\dagger}, N(\%)$ & & & \\
Atrial fibrillation, $N(\%)$ & $1(8.3 \%)$ & $1(2.2 \%)$ & 0.380 \\
CCl & $2.5(1.3-3.8)$ & $2.0(2.0-3.0)$ & 0.928 \\
Antiplatelet use, $N(\%)$ & $7(58.3 \%)$ & $24(53.3 \%)$ & 1.000 \\
Anticoagulant use, $N$ & $1(8.3 \%)$ & $1(2.2 \%)$ & 0.380 \\
(\%) & & & \\
\hline
\end{tabular}

$\mathrm{ICH}$, intracerebral hemorrhage; $\mathrm{CCl}$, Charlson comorbidity index.

Continuous variables, presented as median values and interquartile ranges (IQRs), were analyzed with the Mann-Whitney U-test; categorical variables, presented as number of patients with percentage, were examined with the Chi-square test.

†Includes: peripheral artery disease, coronary artery disease, and carotid artery disease.

dementia diagnosis, we developed a logistic regression model after adjustment for age, sex, and CCI. Adjusted odds ratio (OR) values are reported with $95 \%$ CIs.

\section{RESULTS}

\section{Demographic and Imaging Characteristics of the Entire Cohort}

Fifty-seven patients with multiple lobar CMBs were divided into dementia group $(N=12)$ and non-dementia group $(N=45)$. The two groups differed significantly with respect to subcortical and total ARWMC scale score, CAA-SVD score, $\mathrm{C}_{1}$ score, and $\mathrm{C}_{2}$ score (Tables 1, 2).

\section{Correlations of Imaging Variables Within Variable Categories of the Entire Cohort}

Most MARS and ARWMC scale items showed fair correlation with each other and moderate-to-strong correlation with CAASVD score, $\mathrm{C}_{1}$ score, and $\mathrm{C}_{2}$ score (Table 3). PVSE did not correlate with any other items except $\mathrm{C}_{2}$ score. ICH amount fairly correlated with CAA-SVD score and $\mathrm{C}_{1}$ score, while lacune burden fairly correlated with $\mathrm{C}_{2}$ score (Table 3 ).

\section{Correlations of Imaging Variables With Cognitive Measures of the Dementia Group} Infratentorial and deep MARS scales moderately-to-strongly negatively correlated with short-term memory (registration and recall) and visuoexecutive function, lobar MARS scale fairly with language, and total MARS scale moderately with language and visuoexecutive function (Table 4). BG ARWMC scale moderately-to-strongly negatively correlated with global cognition (MoCA) and language, while subcortical and total ARWMC scales moderately-to-strongly negatively correlated
TABLE 2 | Between-group comparison of imaging variables, $N=57$.

\begin{tabular}{|c|c|c|c|}
\hline Variable & Dementia & Non-dementia & $P$ \\
\hline \multicolumn{4}{|l|}{ MARS } \\
\hline Infratentorial & $4.0(0.0-10.5)$ & $1.0(0.0-8.0)$ & 0.545 \\
\hline Deep & $4.5(0.25-32.0)$ & $2.5(0.0-13.75)$ & 0.423 \\
\hline Lobar & $30.50(4.3-55.0)$ & $5.5(2.0-22.25)$ & 0.105 \\
\hline Total & $32.5(7.5-88.0)$ & $11.5(3.0-39.75)$ & 0.119 \\
\hline Cortical superficial siderosis & $6(50.0 \%)$ & $10(22.2 \%)$ & 0.076 \\
\hline \multicolumn{4}{|l|}{ ARWMC } \\
\hline Infratentorial & $0.0(0.0-0.0)$ & $0.0(0.0-0.5)$ & 0.572 \\
\hline Basal ganglia & $2.0(1.0-2.0)$ & $2.0(1.0-2.0)$ & 0.299 \\
\hline Subcortical & $6.0(5.0-7.0)$ & $4.0(3.0-5.5)$ & $0.003^{\star}$ \\
\hline Total & $9.5(8.0-11.75)$ & $8.0(4.0-10.0)$ & $0.015^{\star}$ \\
\hline \multicolumn{4}{|l|}{ PVSE } \\
\hline Centrum semiovale & $3.0(2.0-3.75)$ & $3.0(2.5-4.0)$ & 0.383 \\
\hline Basal ganglia & $3.0(2.0-3.0)$ & $3.0(2.0-3.0)$ & 0.644 \\
\hline \multicolumn{4}{|l|}{ Lesion quantity } \\
\hline $\mathrm{ICH}$ & $2.0(0.25-3.5)$ & $2.0(0-4.0)$ & 0.795 \\
\hline Lacune & $8.0(3.25-11.75)$ & $3.0(2.0-6.5)$ & 0.05 \\
\hline \multicolumn{4}{|l|}{ Lesion burden score } \\
\hline CAA-SVD score & $4.0(3.25-5.0)$ & $3.0(2.0-4.0)$ & $0.027^{\star}$ \\
\hline $\mathrm{C}_{1}^{\dagger}$ & $11.5(9.25-13.75)$ & $9.0(6.0-11.5)$ & $0.008^{\star \star}$ \\
\hline $\mathrm{C}_{2}^{\ddagger}$ & $13.0(11.0-14.75)$ & $10.0(7.0-13.0)$ & $0.007^{\star \star}$ \\
\hline
\end{tabular}

MARS, Microbleed anatomical rating scale; ARWMC, Age-related white matter change; PVSE, enlargement of the perivascular space; CAA, cerebral amyloid angiopathy; SVD, small vessel disease.

Continuous variables, presented as median (interquartile ranges), subjected to MannWhitney U-test; Categorical variables, presented as the number of patients (percentage) subjected Chi-square test; ${ }^{*} p<0.05,{ }^{*} p<0.01$.

† Sum of total MARS score and total ARWMC scale.

¥ Sum of total MARS score, total ARWMC scale, BG PVSE ( $\geq 20)$, and lacune amount ( $\geq 5)$.

with performance in global function (MMSE and MoCA), short-term memory (recall), orientation, and language (Table 4). Regarding the composite scores, $\mathrm{C}_{1}$ score outperformed CAASVD score and $\mathrm{C}_{2}$ score in the cognitive correlation (Table 4). $\mathrm{C}_{1}$ score moderately-to-strongly negatively correlated with global function, short-term memory (recall), orientation, language, and visuoexecutive function (Table 4).

\section{Associations of Imaging Variables With Dementia Diagnosis}

A logistic regression controlled for age, sex, and CCI (Table 5) revealed positive associations of subcortical ARWMC (OR 2.03; 95\% CI, 1.24-3.32, $p=0.005$ ), total ARWMC (OR 1.43; 95\% CI, $1.09-1.89, p=0.011$ ), lacune number (OR $1.18 ; 95 \% \mathrm{CI}$, $1.02-1.35, p=0.023$ ), CAA-SVD score (OR 2.33; 95\% CI, $1.01-$ $5.40, p=0.047), \mathrm{C}_{1}$ (OR 1.41; 95\% CI, 1.09-1.83, $p=0.009$ ), and $\mathrm{C}_{2}$ (OR 1.38; 95\% CI 1.08-1.76, $p=0.010$ ) with subcortical vascular dementia.

\section{DISCUSSIONS}

This retrospective cohort study demonstrated that subcortical and total WMH burdens and the three composite scores were higher in patients with subcortical vascular dementia than in 
TABLE 3 | Correlations of individual imaging variables of the cohort, $N=57$

\begin{tabular}{|c|c|c|c|c|c|c|c|c|c|c|c|c|c|c|c|c|}
\hline \multirow[b]{2}{*}{ Factors } & & \multicolumn{4}{|c|}{ MARS } & \multicolumn{4}{|c|}{ ARWMC } & \multicolumn{2}{|c|}{ PVSE } & \multicolumn{2}{|c|}{ Lesion quantity } & \multicolumn{3}{|c|}{ Lesion burden score } \\
\hline & & Infratentorial & Deep & Lobar & Total & Infratentorial & BG & Subcortical & Total & cs & BG & $\mathrm{ICH}$ & Lacune & CAA-SVD & $\mathrm{C}_{1}$ & $\mathrm{C}_{2}$ \\
\hline \multirow[t]{3}{*}{ MARS } & Infratentorial & - & & & & & & & & & & & & & & \\
\hline & Deep & $0.734^{\star \star}$ & - & & & & & & & & & & & & & \\
\hline & Lobar & $0.659^{* \star}$ & $0.603^{* \star}$ & - & & & & & & & & & & & & \\
\hline \multirow[t]{3}{*}{ ARWMC } & Infratentorial & $0.306^{*}$ & $0.298^{*}$ & 0.228 & 0.259 & - & & & & & & & & & & \\
\hline & $B G$ & $0.334^{*}$ & 0.205 & $0.288^{*}$ & $0.338^{*}$ & -0.008 & - & & & & & & & & & \\
\hline & Subcortical & $0.423^{* \star}$ & 0.247 & $0.492^{* *}$ & $0.484^{\star \star}$ & $0.272^{*}$ & $0.392^{* *}$ & - & & & & & & & & \\
\hline \multirow[t]{2}{*}{ PVSE } & CS & 0.059 & -0.025 & -0.083 & -0.059 & 0.058 & -0.113 & -0.179 & -0.164 & - & & & & & & \\
\hline & $B G$ & -0.060 & 0.010 & -0.079 & -0.101 & -0.106 & 0.135 & -0.034 & 0.011 & 0.224 & - & & & & & \\
\hline \multirow[t]{2}{*}{ Lesion quantity } & $\mathrm{ICH}$ & $0.369^{* *}$ & $0.373^{* *}$ & 0.162 & $0.287^{\star}$ & 0.205 & 0.110 & 0.225 & 0.250 & 0.112 & -0.159 & - & & & & \\
\hline & Lacune & $0.283^{*}$ & $0.374^{\star \star}$ & 0.247 & $0.295^{\star}$ & 0.148 & 0.258 & 0.244 & $0.302^{*}$ & -0.034 & -0.166 & -0.026 & - & & & \\
\hline \multirow[t]{3}{*}{ Lesion burden score } & CAA-SVD & $0.541^{\star \star}$ & $0.537^{\star *}$ & $0.773^{\star \star}$ & $0.755^{\star *}$ & 0.183 & $0.307^{*}$ & $0.507^{\star \star}$ & $0.558^{\star \star}$ & 0.081 & 0.125 & $0.305^{\star}$ & 0.231 & - & & \\
\hline & $\mathrm{C}_{1}$ & $0.723^{* \star}$ & $0.557^{\star \star}$ & $0.796^{\star \star}$ & $0.819^{* *}$ & $0.370^{* *}$ & $0.513^{\star \star}$ & $0.787^{\star \star}$ & $0.849^{* \star}$ & -0.167 & -0.036 & $0.284^{*}$ & 0.238 & $0.684^{\star \star}$ & - & \\
\hline & $\mathrm{C}_{2}$ & $0.625^{* *}$ & $0.566^{\star \star}$ & $0.574^{* *}$ & $0.622^{* *}$ & $0.346^{* *}$ & $0.443^{* *}$ & $0.508^{* \star}$ & $0.602^{* *}$ & $0.372^{* *}$ & $0.395^{\star \star}$ & 0.199 & $0.425^{\star *}$ & $0.619^{* *}$ & $0.682^{\star *}$ & - \\
\hline
\end{tabular}

ARWMC, Age-related white matter change; BG, basal ganglia; CS, centrum semiovale; ICH, intracerebral hemorrhage; MARS, Microbleed anatomical rating scale; PVSE, enlargement of the perivascular space; CAA, cerebral amyloid angiopathy; SVD, small vessel disease.

Spearman's rank correlation coefficient was calculated to explore correlations between variables. Dark gray block: moderate-to-strong correlation, $0.6 \leq\left|r_{s}\right|$. Light gray block: fair correlation, $0.3 \leq\left|r_{s}\right|<0.6 .{ }^{*} p<0.05,{ }^{* *} p<0.01$.

TABLE 4 | Correlations of individual imaging variables with neurocognitive domains of the dementia group, $N=12$

\begin{tabular}{|c|c|c|c|c|c|c|c|c|c|}
\hline & & MMSE & MoCA & Attention & STM (registration) & STM (recall) & Orientation & Language & Visuoexecutive function \\
\hline \multirow[t]{4}{*}{ MARS } & Infratentorial & -0.493 & -0.540 & -0.239 & $-0.582^{*}$ & $-0.642^{\star}$ & -0.440 & -0.486 & $-0.731^{\star *}$ \\
\hline & Deep & -0.376 & -0.271 & -0.026 & $-0.801^{* *}$ & -0.524 & -0.400 & -0.278 & $-0.627^{\star}$ \\
\hline & Lobar & -0.410 & -0.449 & -0.300 & -0.289 & -0.417 & -0.416 & $-0.583^{*}$ & -0.480 \\
\hline & Total & -0.466 & -0.561 & -0.321 & -0.423 & -0.528 & -0.463 & $-0.650^{*}$ & $-0.661^{*}$ \\
\hline \multirow[t]{4}{*}{ ARWMC } & Infratentorial & -0.033 & 0.001 & 0.002 & 0.098 & -0.299 & -0.066 & 0.131 & 0.073 \\
\hline & Basal ganglia & -0.370 & $-0.601^{*}$ & -0.423 & -0.181 & -0.435 & -0.278 & $-0.664^{*}$ & -0.438 \\
\hline & Subcortical & $-0.675^{*}$ & $-0.665^{\star}$ & -0.574 & -0.022 & $-0.677^{*}$ & $-0.621^{*}$ & $-0.648^{*}$ & -0.541 \\
\hline & Total & $-0.674^{*}$ & $-0.730^{\star *}$ & $-0.614^{*}$ & 0.002 & $-0.716^{\star *}$ & $-0.619^{*}$ & $-0.692^{*}$ & -0.511 \\
\hline \multirow[t]{2}{*}{ PVSE } & CS & 0.329 & 0.080 & -0.026 & 0.319 & 0.390 & 0.427 & 0.095 & 0.366 \\
\hline & $B G$ & -0.131 & -0.326 & -0.231 & 0.246 & -0.263 & -0.074 & -0.285 & -0.052 \\
\hline \multirow[t]{2}{*}{ Lesion quantity } & $\mathrm{ICH}$ & -0.231 & 0.200 & 0.094 & $-0.695^{*}$ & -0.172 & -0.216 & 0.265 & -0.328 \\
\hline & Lacune & 0.002 & 0.056 & 0.307 & -0.452 & -0.365 & -0.023 & 0.179 & -0.079 \\
\hline \multirow[t]{3}{*}{ Lesion burden score } & CAA-SVD & -0.133 & -0.303 & -0.236 & 0.163 & -0.192 & -0.116 & -0.414 & -0.093 \\
\hline & $\mathrm{C} 1$ & $-0.675^{*}$ & $-0.744^{\star \star}$ & -0.563 & -0.190 & $-0.733^{* \star}$ & $-0.660^{*}$ & $-0.753^{\star \star}$ & $-0.606^{*}$ \\
\hline & C2 & -0.336 & -0.575 & -0.351 & -0.134 & $-0.600^{*}$ & -0.233 & -0.502 & -0.315 \\
\hline
\end{tabular}

MMSE, Mini-Mental State Examination; MoCA, Montreal Cognitive Assessment; STM, short-term memory; ARWMC, Age-related white matter change; MARS, Microbleed anatomical rating scale; PVSE, enlargement of the perivascular space; CS, centrum semiovale; BG, basal ganglia; ICH, intracerebral hemorrhage; CAA, cerebral amyloid angiopathy; SVD, small vessel disease.

Spearman's rank correlation coefficient was calculated to explore correlations between variables. Dark gray block: moderate-to-strong correlation, $0.6 \leq\left|r_{s}\right|$. Light gray block: fair correlation, $0.3 \leq\left|r_{s}\right|<0.6 .{ }^{*} p<0.05,{ }^{* *} p<0.01$. 
TABLE 5 | Associations of imaging variables with subcortical vascular dementia, $N=57$.

\begin{tabular}{|c|c|c|}
\hline & \multicolumn{2}{|c|}{ Dementia } \\
\hline & OR $(95 \% \mathrm{Cl})$ & $p$ \\
\hline \multicolumn{3}{|l|}{ MARS } \\
\hline Infratentorial & $1.00(0.93-1.07)$ & 0.963 \\
\hline Deep & 1.03 (0.99-1.08) & 0.161 \\
\hline Lobar & $1.00(0.99-1.02)$ & 0.739 \\
\hline Total & $1.00(0.99-1.01)$ & 0.580 \\
\hline \multicolumn{3}{|l|}{ ARWMC } \\
\hline Infratentorial & $0.53(0.10-2.80)$ & 0.458 \\
\hline Basal ganglia & $1.67(0.74-3.78)$ & 0.221 \\
\hline Subcortical & $2.03(1.24-3.32)$ & $0.005^{\star}$ \\
\hline Total & $1.43(1.09-1.89)$ & $0.011^{*}$ \\
\hline \multicolumn{3}{|l|}{ PVSE } \\
\hline Centrum semiovale & $0.72(0.36-1.42)$ & 0.339 \\
\hline Basal ganglia & $1.10(0.44-2.74)$ & 0.837 \\
\hline \multicolumn{3}{|l|}{ Lesion quantity } \\
\hline $\mathrm{ICH}$ & $1.00(0.75-1.34)$ & 1.000 \\
\hline Lacune & $1.18(1.02-1.35)$ & $0.023^{*}$ \\
\hline \multicolumn{3}{|l|}{ Lesion burden score } \\
\hline CAA-SVD score & $2.33(1.01-5.40)$ & $0.047^{*}$ \\
\hline $\mathrm{C}^{\dagger}$ & $1.41(1.09-1.83)$ & $0.009^{*}$ \\
\hline $\mathrm{C} 2^{\ddagger}$ & $1.38(1.08-1.76)$ & $0.010^{*}$ \\
\hline
\end{tabular}

$I C H$, intracerebral hemorrhage; MARS, Microbleed anatomical rating scale; ARWMC, Age-related White Matter Change; PVSE, enlargement of the perivascular space; CAA, cerebral amyloid angiopathy; SVD, small vessel disease; OR, odd's ratio.

ORs determined by multivariate logistic regression, adjusted for age, sex, and CCl; ${ }^{*} p<0.05$.

† Sum of total MARS score and total ARWMC scale.

$\ddagger$ Sum of total MARS score, total ARWMC scale, BG PVSE $(\geq 20)$, and lacune $(\geq 5)$.

those without dementia. Although most regional measures of CMBs and WMHs correlated directly with one another, only subcortical and total $\mathrm{WMH}$ variables yielded desirable cognitive correlations. PVS ratings did not correlate with other imaging measures or any cognitive scores. Of the three composite scores, only $\mathrm{C}_{1}$ score correlated well with cognitive performance in terms of domain functioning. Of the imaging variables assessed, after adjusting for age, sex, and CCI, WMH load, lacune number, and all composite scores were associated with risk of subcortical vascular dementia. Collectively, these findings suggest that WMH load is more important in determining cognitive consequences than CMB burden and PVSE amount, even though accumulating imaging markers corresponding to CAA pathology (CAA-SVD score) or global SVD burden $\left(C_{1}\right.$ score and $C_{2}$ score) contribute to dementia. Till date, this is the first study to examine the cognitive associations of comprehensive SVD imaging markers, regionally and totally as well as singularly and compositely, in the circumstance of advanced SVD of CAA and HA co-pathologies.

The enrolled subjects with multiple lobar CMBs had a > $80 \%$ prevalence of hypertension history, high CAA-SVD scores reflexive of heavy CAA pathological loads, and a $>80 \%$ prevalence of mixed CMBs, and consequently, advanced HA and
CAA co-pathologies indeed existed in our cohort. We believe that the subjects included in our study belong to an intermediate between SVD type 3 and 4 in the SVD imaging spectrum (43). Although mixed CMBs are considered to be driven mostly by HA, both HA and CAA can cause cerebral hypoperfusion and enhance amyloid production (44), explaining the synergistic effects of HA (Hypertensive Angiopathy) and CAA on the cumulative changes in the vasculopathic and neurodegenerative processes in cognitive deterioration $(8,45)$.

Cerebral microbleeds and WMHs are known to be associated with each other in adults over 40 years old in the general population (46). In our cohort characterized by heavy CMB loads and a high prevalence of hypertension, $\mathrm{CMB}$ burden correlated strongly with WMH load, possibly supporting the notion that SVD might be a mediator of a complex relationship between CMBs and WMHs, both of which are well-established SVD manifestations. Vasculopathic changes implicated in both HA and CAA contexts make the relationship between CMBs and WMHs one of mutual promotion. These shared pathophysiologic characteristics of CMBs and WMHs provide putative mechanistic links.

In concordance with previous findings, lacunes were associated with deep CMBs (which are related to hypertension), but not with lobar CMBs (47). We found that ICH quantity correlates with non-lobar CMBs, rather than lobar CMBs. One previous study demonstrated that patients with mixed-location ICH/CMBs, regardless of the location of the symptomatic bleed, have relatively homogeneous clinical and radiologic profiles similar to patients with hypertensive $\mathrm{ICH}$, rather than those with CAA-related ICH (48). This finding is probably attributable to a substantial presence of traditional vascular risk factors and evidence for hypertensive target-organ damage (48). Together with our results in a population with severe $\mathrm{HA}$ and CAA co-pathologies, a pronounced vascular risk factor burden may be the main driving force for microangiopathy as the major underlying SVD.

Enlargements of the perivascular space have been shown to be associated with other SVD markers, such as WMHs, lacunes, and CMBs, in discordance with our findings of PVSEs not associating with SVD-related markers $(18,49,50)$. The lack of PVSE association could be partially explained by the high prevalence rates of severe CS PVSE (72.1\%) and BG PVSE $(62.3 \%)$ in our entire cohort (51).

Regarding the cognitive correlations of CMBs in our dementia group, deep and infratentorial CMBs correlated with short-term memory decline and visuoexecutive dysfunction, lobar $\mathrm{CMBs}$ with language impairment, and total CMBs with deficits in language and visuoexecutive function. CMB distributions greatly impact cognitive functions $(52,53)$. In previous studies, lobar CMBs correlated with impaired global cognition, attention, and visual executive function, BG CMBs with subnormal global cognition, and infratentorial CMBs with declined language ability and functional status (5255). CMBs might reflect early neurodegenerative processes in a common pathogenesis of cognitive impairment, even though findings concerning effects of $\mathrm{CMB}$ location on cognition are still a matter of debate (56). Amyloid-related 
pathology and HA were shown to produce combined effects on the longitudinal progression of lobar CMBs and decline in attention and visuoexecutive function (13). As a result, it is possible that, in the context of multiple lobar CMBs (implying CAA), non-lobar CMBs (implying HA) may promote a synergism of CAA and $\mathrm{HA}$ in the development of cognitive impairment (13).

With respect to the cognitive associations of WMHs in our dementia group, subcortical, and total WMH burdens were significantly relevant to all cognitive domains, except memory registration and visuoexecutive function, while BG WMHs were related to global cognitive dysfunction and defective language, in line with numerous studies showing the associations of domain-specific cognitive profiles with $\mathrm{WMH}$ topographic changes (57-61). WMH distributions and loads are considered as predictors of cognitive impairment. Subcortical WMH scores in our study stood for the total WMH burdens in the frontal, parieto-occipital, temporal lobes, and regions adjacent to ventricle wall, which are believed to primarily interrupt both short and long connections to spatially distant brain areas. Therefore, subcortical WMHs can cause cognitive decline in multiple domains supported by the specific brain regions. In addition, cognitive dysfunction in vascular dementia may be the consequence of disconnection of the fronto-striatal circuits $(62,63)$. Cognitive tests involving language abilities were strongly related to damage to the fronto-subcortical connections, which are critical for both verbal abilities and global function (64-66). Our findings, along with previous literature, suggest that BG engages in global cognitive functioning contributing to performance in language tasks. We consider that WMHs considerably impact cognition and play a key role in determining the cognitive consequences.

No cognitive associations with regional PVSEs were identified in our dementia group. Advancing age and increasing burdens of CMBs and WMHs may obliterate the PVSE effect on cognition. Previous studies on the PVSE association with cognitive domain-specific analyses demonstrated discrepant findings: no PVSEs in any brain region associated with any cognitive domains, BG PVSEs associated with worse visuomotor speed, PVSEs in the hippocampus linked to better memory, and total PVSEs related to lower global cognition, non-verbal reasoning, and visuoexecutive function $(18,67-$ 72). These inconsistent results imply insufficient evidence connecting either total or regional PVSEs with declines in specific cognitive domains.

The traditional global SVD index, derived by summing the aforementioned four established SVD MRI markers, can predict prognosis but is not feasible in our study (73). CAA-SVD, $\mathrm{C}_{1}$, and $\mathrm{C}_{2}$ scores were higher in our dementia group than in non-dementia group. Only $\mathrm{C}_{1}$ score significantly correlated with impairment in global cognition, recall memory, orientation, language, and visuoexecutive function as compared with CAASVD score (representative of entire CAA co-pathological burden) and $\mathrm{C}_{2}$ score (indicative of global SVD imaging burden). Unlike previous study showing an association of CAASVD score with cognitive functions, the discrepant finding may be attributed to varying population characteristics (74).
Combining measures of total $\mathrm{CMBs}$ and WMHs (i.e., $\mathrm{C}_{1}$ score) added further correlation coefficients in the cognitive correlation analysis in contrast to regional and total imaging scores of CMBs or WMHs alone. Hence, we consider that incorporating parameters of $\mathrm{CMBs}$ and $\mathrm{WMHs}$ (i.e., $\mathrm{C}_{1}$ score), instead of global SVD imaging burden score (i.e., $\mathrm{C}_{2}$ score), is a potential imaging surrogate for domain-related cognitive impairment. Likewise, the entire CAA pathological burden score (i.e., CAA-SVD scoring) did not essentially correspond to domain-specific cognitive decline, specifically in a population with high burden of mixed pathologies in our study, which may undermine the cognitive associations of CAASVD scoring.

Of the imaging variables examined in this study, higher subcortical and total WMH loads, lacune number, CAA-SVD scores, $\mathrm{C}_{1}$ scores, and $\mathrm{C}_{2}$ scores posed increased risk of subcortical vascular dementia. CMBs, WMHs, and lacunes are well-described decisive imaging correlates of cognitive impairment and dementia of vascular and neurodegenerative processes $(45,75,76)$. Accumulating imaging correspondences of CAA pathology (CAA-SVD score) and global SVD load ( $\mathrm{C}_{1}$ score and $\mathrm{C}_{2}$ score) were consistently risky for dementia diagnosis. However, despite being a prevalent imaging finding in SVD, PVSEs have not expressed invariable predictability for cognitive decline. MARS scoring system that quantifies CMBs through numerical value, rather than on an ordinal scale, may account for no association of distributional CMB burdens with dementia risk in our study. Combining $\mathrm{CMB}$ and $\mathrm{WMH}$ markers (i.e., $\mathrm{C}_{1}$ score) reflective of two distinct underlying pathophysiological processes in CAA and HA did indeed show an incremental cognitive correlation as compared with CAA-SVD score and $\mathrm{C}_{2}$ score.

This study had several limitations. First, this study included a relatively small population from a single center. A larger cohort with longitudinal follow-up is necessary to clarify these findings and their reproducibility. Second, we did not use volumetric analysis and visual ratings may not precisely represent the entire lesion burden, but they are relatively easy to do and are readily applicable in clinical practice. Third, we did not analyze lobe-specific cognitive functions and distributions of individual imaging variables. Fourth, we did not analyze cortical microinfarcts, although some studies reported significant association between cognitive decline and cortical microbleeds. Finally, the cognitive associations of SVD imaging markers of the participants recruited from our stroke registry were not examined because comprehensive cognitive assessments were not routinely performed for these patients. This might account for some medium-sized but non-significant associations in our results.

In conclusion, individual imaging markers correlated differentially with one another and had differing relationships with cognitive domains. WMH burden seems to be the major imaging correlate of deficits across extensive cognitive domains. The present findings buttress previous research demonstrating $\mathrm{HA}$ and CAA co-pathologies contribute synergistically to the pathophysiological progression of cognitive decline in severe SVD. 


\section{DATA AVAILABILITY STATEMENT}

The original contributions presented in the study are included in the article/supplementary material, further inquiries can be directed to the corresponding author/s.

\section{ETHICS STATEMENT}

The studies involving human participants were reviewed and approved by Taichung Veterans General Hospital. Written informed consent for participation was not required for this study in accordance with the national legislation and the institutional requirements.

\section{REFERENCES}

1. Pantoni L. Cerebral small vessel disease: from pathogenesis and clinical characteristics to therapeutic challenges. Lancet Neurol. (2010) 9:689-701. doi: 10.1016/S1474-4422(10)70104-6

2. Brown R, Benveniste H, Black SE, Charpak S, Dichgans M, Joutel A, et al. Understanding the role of the perivascular space in cerebral small vessel disease. Cardiovasc Res. (2018) 114:1462-73. doi: 10.1093/cvr/cvy113

3. Cuadrado-Godia E, Dwivedi P, Sharma S, Ois Santiago A, Roquer Gonzalez J, Balcells $\mathrm{M}$, et al. Cerebral small vessel disease: a review focusing on pathophysiology, biomarkers, and machine learning strategies. J Stroke. (2018) 20:302-20. doi: 10.5853/jos.2017.02922

4. Charidimou A, Martinez-Ramirez S, Reijmer YD, Oliveira-Filho J, Lauer A, Roongpiboonsopit $\mathrm{D}$, et al. Total magnetic resonance imaging burden of small vessel disease in cerebral amyloid angiopathy: an imagingpathologic study of concept validation. JAMA Neurol. (2016) 73:994-1001. doi: 10.1001/jamaneurol.2016.0832

5. Amin Al Olama A, Wason JMS, Tuladhar AM, van Leijsen EMC, Koini M, Hofer E, et al. Simple MRI score aids prediction of dementia in cerebral small vessel disease. Neurology. (2020) 94:e1294-302. doi: 10.1212/WNL.0000000000009141

6. Pinter D, Ritchie SJ, Doubal F, Gattringer T, Morris Z, Bastin ME, et al. Impact of small vessel disease in the brain on gait and balance. Sci Rep. (2017) 7:41637. doi: $10.1038 /$ srep41637

7. Staals J, Booth T, Morris Z, Bastin ME, Gow AJ, Corley J, et al. Total MRI load of cerebral small vessel disease and cognitive ability in older people. Neurobiol Aging. (2015) 36:2806-11. doi: 10.1016/j.neurobiolaging.2015. 06.024

8. Xiong L, Boulouis G, Charidimou A, Roongpiboonsopit D, Jessel MJ, Pasi $\mathrm{M}$, et al. Dementia incidence and predictors in cerebral amyloid angiopathy patients without intracerebral hemorrhage. J Cereb Blood Flow Metab. (2018) 38:241-9. doi: 10.1177/0271678X17700435

9. Kim BJ, Lee S-H. Cerebral microbleeds: their associated factors, radiologic findings, and clinical implications. J Stroke. (2013) 15:153-63. doi: 10.5853/jos.2013.15.3.153

10. Puy L, Pasi M, Rodrigues M, van Veluw SJ, Tsivgoulis G, Shoamanesh A, et al. Cerebral microbleeds: from depiction to interpretation. J Neurol Neurosurg Psychiatry. (2021). doi: 10.1136/jnnp-2020-323951

11. Schreiber S, Wilisch-Neumann A, Schreiber F, Assmann A, Scheumann $\mathrm{V}$, Perosa V, et al. Invited review: the spectrum of age-related small vessel diseases: potential overlap and interactions of amyloid and nonamyloid vasculopathies. Neuropathol Appl Neurobiol. (2020) 46:219-39. doi: 10.1111/nan.12576

12. Poels MMF, Mesker DJ, Ikram MA, Vernooij MW, Hofman A, Vrooman HA, et al. Lobar distribution of cerebral microbleeds: the Rotterdam Scan Study. JALZ Alzheimer's Dementia. (2009) 5:P32-P. doi: 10.1016/j.jalz.2009. 05.622
AUTHOR CONTRIBUTIONS

T-BC conceptualized and designed the study, analyzed the data, and did the critical revision of the manuscript. C-YL analyzed the imaging data, performed the statistical analysis, and wrote the manuscript. H-CC and S-RJ contributed to the acquisition and interpretation of the imaging data. T-BC, $\mathrm{W}-\mathrm{JL}$, and P-LC contributed to patient collection. J-PC performed the statistical analysis. All authors read and approved the submitted version.

\section{FUNDING}

We are deeply indebted to Taichung Veterans General Hospital for providing the grants for this study (TCVGH-1103401A).

13. Kim YJ, Kim HJ, Park JH, Kim S, Woo SY, Kwak KC, et al. Synergistic effects of longitudinal amyloid and vascular changes on lobar microbleeds. Neurology. (2016) 87:1575-82. doi: 10.1212/WNL.0000000000003220

14. Merino JG. White matter hyperintensities on magnetic resonance imaging: what is a clinician to do? Mayo Clin Proc. (2019) 94:380-2. doi: 10.1016/j.mayocp.2019.01.016

15. Rost NS, Etherton M. Cerebral small vessel disease. Continuum. (2020) 26:332-52. doi: 10.1212/CON.0000000000000841

16. Prins ND, Scheltens $\mathrm{P}$. White matter hyperintensities, cognitive impairment and dementia: an update. Nat Rev Neurol. (2015) 11:157-65. doi: 10.1038/nrneurol.2015.10

17. Wardlaw JM, Benveniste H, Nedergaard M, Zlokovic BV, Mestre H, Lee H, et al. Perivascular spaces in the brain: anatomy, physiology and pathology. Nat Rev Neurol. (2020) 16:137-53. doi: 10.1038/s41582-020-0312-z

18. Francis F, Ballerini L, Wardlaw JM. Perivascular spaces and their associations with risk factors, clinical disorders and neuroimaging features: a systematic review and meta-analysis. Int J Stroke. (2019) 14:359-71. doi: $10.1177 / 1747493019830321$

19. Yakushiji Y, Charidimou A, Hara M, Noguchi T, Nishihara M, Eriguchi $\mathrm{M}$, et al. Topography and associations of perivascular spaces in healthy adults: the Kashima scan study. Neurology. (2014) 83:2116-23. doi: 10.1212/WNL.0000000000001054

20. Pasi M, Sugita L, Xiong L, Charidimou A, Boulouis G, Pongpitakmetha $\mathrm{T}$, et al. Association of cerebral small vessel disease and cognitive decline after intracerebral hemorrhage. Neurology. (2021) 96:e182-e92. doi: 10.1212/WNL.0000000000011050

21. Charlson ME, Pompei P, Ales KL, MacKenzie CR. A new method of classifying prognostic comorbidity in longitudinal studies: development and validation. J Chronic Dis. (1987) 40:373-83. doi: 10.1016/0021-9681(87)90171-8

22. Vakil E, Blachstein H. Rey auditory-verbal learning test: structure analysis. J Clin Psychol. (1993) 49:883-90. doi: 10.1002/10974679(199311)49:6<883::AID-JCLP2270490616>3.0.CO;2-6

23. Vanderploeg $\mathrm{RD}$, Schinka JA, Jones $\mathrm{T}$, Small BJ, Graves AB, Mortimer JA. Elderly norms for the Hopkins Verbal Learning Test-Revised. Clin Neuropsychol. (2000) 14:318-24. doi: 10.1076/1385-4046(200008)14:3;1-P;FT318

24. Teng EL, Hasegawa K, Homma A, Imai Y, Larson E, Graves A, et al. The Cognitive Abilities Screening Instrument (CASI): a practical test for cross-cultural epidemiological studies of dementia. Int Psychogeriatr. (1994) 6:45-58; discussion 62. doi: 10.1017/S1041610294001602

25. Chiu EC, Chien TH, Lee YC. Measurement equivalence between the original and estimated mini-mental state examination in people with dementia. Int J Environ Res Public Health. (2021) 18:7616. doi: 10.3390/ijerph18147616

26. Tsai CF, Lee WJ, Wang SJ, Shia BC, Nasreddine Z, Fuh JL. Psychometrics of the Montreal Cognitive Assessment (MoCA) and its subscales: validation of the Taiwanese version of the MoCA and an item response theory analysis. Int Psychogeriatr. (2012) 24:651-8. doi: 10.1017/S1041610211002298 
27. Hughes CP, Berg L, Danziger WL, Coben LA, Martin RL. A new clinical scale for the staging of dementia. Br J Psychiatry. (1982) 140:566-72. doi: 10.1192/bjp.140.6.566

28. Arvanitakis Z, Shah RC, Bennett DA. Diagnosis and management of dementia: review. JAMA. (2019) 322:1589-99. doi: 10.1001/jama.2019.4782

29. McKhann G, Drachman D, Folstein M, Katzman R, Price D, Stadlan EM. Clinical diagnosis of Alzheimer's disease: report of the NINCDS-ADRDA work group under the auspices of department of health and human services task force on Alzheimer's disease. Neurology. (1984) 34:939-44. doi: 10.1212/WNL.34.7.939

30. McKhann GM, Knopman DS, Chertkow H, Hyman BT, Jack CR, Jr., et al. The diagnosis of dementia due to Alzheimer's disease: recommendations from the National Institute on Aging-Alzheimer's Association workgroups on diagnostic guidelines for Alzheimer's disease. Alzheimer's Dementia. (2011) 7:263-9. doi: 10.1016/j.jalz.2011.03.005

31. Román GC, Tatemichi TK, Erkinjuntti T, Cummings JL, Masdeu JC, Garcia $\mathrm{JH}$, et al. Vascular dementia: diagnostic criteria for research studies. report of the NINDS-AIREN International Workshop. Neurology. (1993) 43:250-60. doi: 10.1212/WNL.43.2.250

32. McKhann GM, Albert MS, Grossman M, Miller B, Dickson D, Trojanowski JQ. Clinical and pathological diagnosis of frontotemporal dementia: report of the Work Group on Frontotemporal Dementia and Pick's Disease. Arch Neurol. (2001) 58:1803-9. doi: 10.1001/archneur.58.11.1803

33. Rascovsky K, Hodges JR, Knopman D, Mendez MF, Kramer JH, Neuhaus J, et al. Sensitivity of revised diagnostic criteria for the behavioural variant of frontotemporal dementia. Brain. (2011) 134:2456-77. doi: 10.1093/brain/awr179

34. McKeith IG, Boeve BF, Dickson DW, Halliday G, Taylor JP, Weintraub D, et al. Diagnosis and management of dementia with Lewy bodies: fourth consensus report of the DLB Consortium. Neurology. (2017) 89:88-100. doi: 10.1212/WNL.0000000000004058

35. Adams HP Jr, Bendixen BH, Kappelle LJ, Biller J, Love BB, Gordon DL, et al. Classification of subtype of acute ischemic stroke. definitions for use in a multicenter clinical trial. TOAST. Trial of Org 10172 in Acute Stroke Treatment. Stroke. (1993) 24:35-41. doi: 10.1161/01.STR.24.1.35

36. Wardlaw JM, Smith EE, Biessels GJ, Cordonnier C, Fazekas F, Frayne R, et al. Neuroimaging standards for research into small vessel disease and its contribution to ageing and neurodegeneration. Lancet Neurol. (2013) 12:822-38. doi: 10.1016/S1474-4422(13)70124-8

37. Gregoire SM, Chaudhary UJ, Brown MM, Yousry TA, Kallis C, Jager $\mathrm{HR}$, et al. The Microbleed Anatomical Rating Scale (MARS): reliability of a tool to map brain microbleeds. Neurology. (2009) 73:1759-66. doi: 10.1212/WNL.0b013e3181c34a7d

38. Wahlund LO, Barkhof F, Fazekas F, Bronge L, Augustin M, Sjögren M, et al. A new rating scale for age-related white matter changes applicable to MRI and CT. Stroke. (2001) 32:1318-22. doi: 10.1161/01.STR.32.6.1318

39. van Straaten EC, Fazekas F, Rostrup E, Scheltens P, Schmidt R, Pantoni $\mathrm{L}$, et al. Impact of white matter hyperintensities scoring method on correlations with clinical data: the LADIS study. Stroke. (2006) 37:836-40. doi: 10.1161/01.STR.0000202585.26325.74

40. Fan YH, Lam WW, Mok VC, Huang RX, Wong KS. Variability and validity of a simple visual rating scale in grading white matter changes on magnetic resonance imaging. J Neuroimaging. (2003) 13:255-8. doi: 10.1111/j.1552-6569.2003.tb00187.x

41. Fazekas F, Chawluk JB, Alavi A, Hurtig HI, Zimmerman RA. MR signal abnormalities at $1.5 \mathrm{~T}$ in Alzheimer's dementia and normal aging. AJR Am J Roentgenol. (1987) 149:351-6. doi: 10.2214/ajr.149.2.351

42. Doubal FN, MacLullich AM, Ferguson KJ, Dennis MS, Wardlaw JM. Enlarged perivascular spaces on MRI are a feature of cerebral small vessel disease. Stroke. (2010) 41:450-4. doi: 10.1161/STROKEAHA.109.564914

43. Chou KH, Lee PL, Peng LN, Lee WJ, Wang PN, Chen LK, et al. Classification differentiates clinical and neuroanatomic features of cerebral small vessel disease. Brain Commun. (2021) 3:fcab107. doi: 10.1093/braincomms/fcab107

44. Thomas T, Miners S, Love S. Post-mortem assessment of hypoperfusion of cerebral cortex in Alzheimer's disease and vascular dementia. Brain. (2015) 138:1059-69. doi: 10.1093/brain/awv025

45. Kim HW, Hong J, Jeon JC. Cerebral small vessel disease and alzheimer's disease: a review. Front Neurol. (2020) 11:927. doi: 10.3389/fneur.2020. 00927
46. Cordonnier C, Al-Shahi Salman R, Wardlaw J. Spontaneous brain microbleeds: systematic review, subgroup analyses and standards for study design and reporting. Brain. (2007) 130:1988-2003. doi: 10.1093/brain/awl387

47. Gao Z, Zhai Y, Zhao X, Wang W, Wu W, Wang Z, et al. Deep cerebral microbleeds are associated with the severity of lacunar infarcts and hypertension: a retrospective analysis. Medicine. (2018) 97:e11031. doi: 10.1097/MD.0000000000011031

48. Pasi M, Charidimou A, Boulouis G, Auriel E, Ayres A, Schwab KM, et al. Mixed-location cerebral hemorrhage/microbleeds: underlying microangiopathy and recurrence risk. Neurology. (2018) 90:e119-26. doi: 10.1212/WNL.0000000000004797

49. Charidimou A, Boulouis G, Pasi M, Auriel E, van Etten ES, Haley K, et al. MRI-visible perivascular spaces in cerebral amyloid angiopathy and hypertensive arteriopathy. Neurology. (2017) 88:1157-64. doi: 10.1212/WNL.0000000000003746

50. Charidimou A, Boulouis G, Haley K, Auriel E, van Etten ES, Fotiadis $\mathrm{P}$, et al. White matter hyperintensity patterns in cerebral amyloid angiopathy and hypertensive arteriopathy. Neurology. (2016) 86:505-11. doi: 10.1212/WNL.0000000000002362

51. Lau K-K, Li L, Lovelock CE, Zamboni G, Chan T-T, Chiang M-F, et al. Clinical correlates, ethnic differences, and prognostic implications of perivascular spaces in transient ischemic attack and ischemic stroke. Stroke. (2017) 48:1470-7. doi: 10.1161/STROKEAHA.117.016694

52. Chung CP, Chou KH, Chen WT, Liu LK, Lee WJ, Chen LK, et al. Strictly lobar cerebral microbleeds are associated with cognitive impairment. Stroke. (2016) 47:2497-502. doi: 10.1161/STROKEAHA.116.014166

53. Yakushiji Y, Noguchi T, Hara M, Nishihara M, Eriguchi M, Nanri Y, et al. Distributional impact of brain microbleeds on global cognitive function in adults without neurological disorder. Stroke. (2012) 43:1800-5. doi: 10.1161/STROKEAHA.111.647065

54. van Es AC, van der Grond J, de Craen AJ, Westendorp RG, Bollen EL, Blauw GJ, et al. Cerebral microbleeds and cognitive functioning in the PROSPER study. Neurology. (2011) 77:1446-52. doi: 10.1212/WNL.0b013e318232abld

55. Poels MM, Ikram MA, van der Lugt A, Hofman A, Niessen WJ, Krestin GP, et al. Cerebral microbleeds are associated with worse cognitive function: the Rotterdam Scan Study. Neurology. (2012) 78:326-33. doi: 10.1212/WNL.0b013e3182452928

56. Martinez-Ramirez S, Greenberg SM, Viswanathan A. Cerebral microbleeds: overview and implications in cognitive impairment. Alzheimers Res Ther. (2014) 6:33. doi: 10.1186/alzrt263

57. Debette S, Markus HS. The clinical importance of white matter hyperintensities on brain magnetic resonance imaging: systematic review and meta-analysis. Bmj. (2010) 341:c3666. doi: 10.1136/bmj.c3666

58. Bolandzadeh N, Davis JC, Tam R, Handy TC, Liu-Ambrose T. The association between cognitive function and white matter lesion location in older adults: a systematic review. BMC Neurol. (2012) 12:126. doi: 10.1186/1471-2377-12-126

59. van den Berg E, Geerlings MI, Biessels GJ, Nederkoorn PJ, Kloppenborg RP. White matter hyperintensities and cognition in mild cognitive impairment and Alzheimer's disease: a domain-specific meta-analysis. J Alzheimers Dis (2018) 63:515-27. doi: 10.3233/JAD-170573

60. Kaskikallio A, Karrasch M, Rinne JO, Tuokkola T, Parkkola R, GrönholmNyman P. Domain-specific cognitive effects of white matter pathology in old age, mild cognitive impairment and Alzheimer's disease. Neuropsychol Dev Cogn B Aging Neuropsychol Cogn. (2020) 27:453-70. doi: 10.1080/13825585.2019.1628916

61. Pantoni L, Poggesi A, Inzitari D. The relation between whitematter lesions and cognition. Curr Opin Neurol. (2007) 20:390-7. doi: 10.1097/WCO.0b013e328172d661

62. Looi JC, Sachdev PS. Vascular dementia as a frontal subcortical system dysfunction. Psychol Med. (2000) 30:997-1003. doi: 10.1017/ S003329179900269X

63. Acharya A, Liang X, Tian W, Jiang C, Han Y, Yi L. White matter hyperintensities relate to basal ganglia functional connectivity and memory performance in aMCI and SVMCI. Front Neurosci. (2019) 13:1204. doi: 10.3389/fnins.2019.01204

64. Fedorenko E, Behr MK, Kanwisher N. Functional specificity for high-level linguistic processing in the human brain. Proc Natl Acad Sci USA. (2011) 108:16428-33. doi: 10.1073/pnas.1112937108 
65. Barbas H, García-Cabezas M, Zikopoulos B. Frontal-thalamic circuits associated with language. Brain Lang. (2013) 126:49-61. doi: 10.1016/j.bandl.2012.10.001

66. Camerino I, Sierpowska J, Reid A, Meyer NH, Tuladhar AM, Kessels RPC, et al. White matter hyperintensities at critical crossroads for executive function and verbal abilities in small vessel disease. Hum Brain Mapp. (2021) 42:993-1002. doi: 10.1002/hbm.25273

67. Hurford R, Charidimou A, Fox Z, Cipolotti L, Jager R, Werring DJ. MRIvisible perivascular spaces: relationship to cognition and small vessel disease MRI markers in ischaemic stroke and TIA. J Neurol Neurosurg Psychiatry. (2014) 85:522-5. doi: 10.1136/jnnp-2013-305815

68. Hilal S, Tan CS, Adams HHH, Habes M, Mok V, Venketasubramanian $\mathrm{N}$, et al. Enlarged perivascular spaces and cognition: a meta-analysis of 5 population-based studies. Neurology. (2018) 91:e832-42. doi: 10.1212/ WNL.0000000000006079

69. Paradise M, Crawford JD, Lam BCP, Wen W, Kochan NA, Makkar $\mathrm{S}$, et al. Association of dilated perivascular spaces with cognitive decline and incident dementia. Neurology. (2021) 96:e1501-11. doi: 10.1212/WNL.0000000000011537

70. Maclullich AM, Wardlaw JM, Ferguson KJ, Starr JM, Seckl JR, Deary IJ. Enlarged perivascular spaces are associated with cognitive function in healthy elderly men. J Neurol Neurosurg Psychiatry. (2004) 75:1519-23. doi: 10.1136/jnnp.2003.030858

71. Benjamin P, Trippier S, Lawrence AJ, Lambert C, Zeestraten E, Williams $\mathrm{OA}$, et al. Lacunar infarcts, but not perivascular spaces, are predictors of cognitive decline in cerebral small-vessel disease. Stroke. (2018) 49:586-93. doi: 10.1161/STROKEAHA.117.017526

72. Zhu YC, Dufouil C, Soumaré A, Mazoyer B, Chabriat H, Tzourio C. High degree of dilated Virchow-Robin spaces on MRI is associated with increased risk of dementia. J Alzheimers Dis. (2010) 22:663-72. doi: 10.3233/JAD-2010-100378

73. Zanon Zotin MC, Sveikata L, Viswanathan A, Yilmaz P. Cerebral small vessel disease and vascular cognitive impairment: from diagnosis to management. Curr Opin Neurol. (2021) 34:246-57. doi: 10.1097/WCO.0000000000000913
74. Matsuda K, Shindo A, Ii Y, Tabei K-i, Ueda Y, Ishikawa H, et al. Investigation of hypertensive arteriopathy-related and cerebral amyloid angiopathy-related small vessel disease scores in patients from a memory clinic: a prospective single-centre study. BMJ Open. (2021) 11:e042550. doi: 10.1136/bmjopen-2020-042550

75. Hu HY, Ou YN, Shen XN, Qu Y, Ma YH, Wang ZT, et al. White matter hyperintensities and risks of cognitive impairment and dementia: a systematic review and meta-analysis of 36 prospective studies. Neurosci Biobehav Rev. (2021) 120:16-27. doi: 10.1016/j.neubiorev.2020. 11.007

76. Wang M, Hu H-Y, Wang Z-T, Ou Y-N, Qu Y, Ma Y-H, et al. Association of cerebral microbleeds with risks of cognitive impairment and dementia: a systematic review and meta-analysis of prospective studies. Brain Disorders. (2021) 2:100010. doi: 10.1016/j.dscb.2021. 100010

Conflict of Interest: The authors declare that the research was conducted in the absence of any commercial or financial relationships that could be construed as a potential conflict of interest.

Publisher's Note: All claims expressed in this article are solely those of the authors and do not necessarily represent those of their affiliated organizations, or those of the publisher, the editors and the reviewers. Any product that may be evaluated in this article, or claim that may be made by its manufacturer, is not guaranteed or endorsed by the publisher.

Copyright (c) 2021 Lin, Jhan, Lee, Chen, Chen, Chen and Chen. This is an open-access article distributed under the terms of the Creative Commons Attribution License (CC $B Y)$. The use, distribution or reproduction in other forums is permitted, provided the original author(s) and the copyright owner(s) are credited and that the original publication in this journal is cited, in accordance with accepted academic practice. No use, distribution or reproduction is permitted which does not comply with these terms. 